\title{
Targeted synthetic disease-modifying antirheumatic drugs in spondyloarthritis
}

\author{
“...targeted synthetic DMARDs evaluated, demonstrated interesting \\ results in some subsets of patients with spondyloarthritis. But, to date, \\ the studies are scarce, and the results need to be confirmed."
}

First draft submitted: 2 January 2017; Accepted for publication: 19 January 2017; Published online: 24 February 2017

Keywords: apremilast $\bullet$ kinase inhibitors $\bullet$ nilotinib $\bullet$ psoriatic arthritis $\bullet$ spondyloarthritis - tofacitinib • treatment $\bullet$ tsDMARD

Spondyloarthritis $(\mathrm{SpA})$ is a chronic inflammatory rheumatic disorder characterized by predominantly axial involvement (spine and sacroiliac joints), with a close relation with genetic background (HLA-B27) [1]. SpA encompasses several subtypes (ankylosing spondylitis with typical radiographic changes in sacroiliac joints, reactive arthritis, inflammatory bowel disease associated arthritis, some forms of psoriatic arthritis) and may be classified in several phenotypic presentations (axial forms, peripheral synovitic forms, peripheral enthesitis forms) [2]. The most recent criteria system allows recognition of nonradiographic axial SpA [1]. Inflammatory involvement of the entheses represents the hallmark of the disease. Some extra rheumatic features are frequently associated with SpA: uveitis, psoriasis, inflammatory bowel disease. The disease begins principally in young adults and may impair functional capacity and work presenteeism/ productivity.

Nonsteroidal anti-inflammatory drugs remain the cornerstone of the pharmacological treatment. In case of inadequate response, disease-modifying antirheumatic drugs (DMARDs) may be prescribed [3]. A new nomenclature of DMARDs has recently been proposed [4], with two main categories: synthetic DMARDs and biological DMARDs. Synthetic DMARDS are classified as conventional synthetic DMARDs (csDMARDs; methotrexate, salazopyrine and leflunomide) and targeted synthetic DMARDs (tsDMARDs). The latter category corresponds to small molecules targeting intracellular transduction pathways.

In $\mathrm{SpA}$, biologic DMARDs approved are the several TNF inhibitors for ankylosing spondylitis and nonradiographic axial SpA, and more recently IL-17A inhibitors (currently secukinumab) for ankylosing spondylitis (axial SpA with radiographic involvement of the sacroiliac joints). In the subgroup of psoriatic arthritis, the possibilities are broader, and besides anti-TNF- $\alpha$ and antiIL-17A agents, ustekinumab-targeting of the p40 subunit common to IL-12 and IL-23 is also approved in this indication.

\section{Small molecules (tsDMARDs) in SpA}

This family of pharmacological compounds comprises several types of agents: phosphodiesterase inhibitors and kinase inhibitors.

Apremilast is a phosphodiesterase- 4 inhibitor, modulates expression of inflammatory mediators and exerts anti-inflammatory activity, decreasing pro-inflammatory cytokines.

This tsDMARD is licensed for the treatment of psoriatic arthritis, based on the results of Phase II and Phase III studies, developed in the peripheral phenotype of this condition $[5,6]$, with benefit upon signs and symptoms of patients with psoriatic arthritis and inadequate response to csDMARDs,

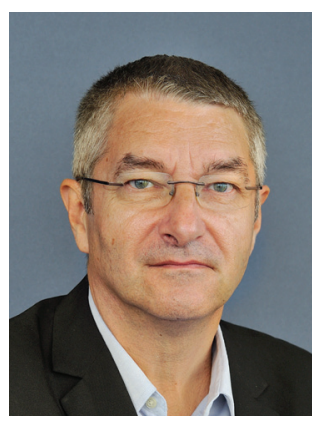

Daniel Wendling * EA 4266, Université de Bourgogne Franche-Comté, Besançon; \&, CHRU de Besançon (University Teaching Hospital), Boulevard Fleming, F-25030, Besançon, France

*Author for correspondence: dwendling@chu-besancon.fr

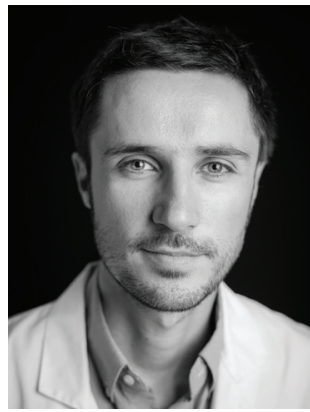

Clément Prati*

EA 4267, FHU INCREASE, Université de Bourgogne Franche-Comté, Besançon; \&, Department of Rheumatology, CHRU de Besançon (University Teaching Hospital), Boulevard Fleming, F-25030, Besançon, France

*Author for correspondence: cprati@chu-besancon.fr

\section{Future $\because$ Medicine part of}


and even in a subgroup of patients with inadequate response to anti-TNF therapy.

In the axial phenotype ankylosing spondylitis, a small controlled study compared over a 3-month period of treatment with apremilast $(\mathrm{n}=17)$ or placebo $(\mathrm{n}=$ 19) and found a decrease in disease activity (BASDAI) in the treatment group compared with placebo [7]. But a prospective controlled trial (POSTURE) comparing two dosages of apremilast (163 patients in each group) or placebo $(\mathrm{n}=164)$ failed to demonstrate any difference at week 16 with placebo using the ASAS 20 outcome measure [8].

Kinase inhibitors represent a large family of small molecules targeting the several types of kinases. The main members of the kinases family are Janus kinases (JAK), spleen tyrosine kinase (SYK), tyrosine kinase (c-kit), Bruton tyrosine kinase, PI3K, P38 MAPK, c-jun terminal kinase, extracellular signal regulated kinase [9]. Many kinase inhibitors are available and under evaluation in several diseases. JAK inhibitors are the most studied in rheumatic diseases, and tofacitinib is approved in the USA for the treatment of rheumatoid arthritis.

Tofacitinib was evaluated in the treatment of psoriatic arthritis in a randomized controlled trial [10] comparing two dosages of tofacitinib $(2 \times 5$ and $2 \times 10$ mg per day) with adalimumab (anti-TNF monoclonal antibody, approved in the treatment of psoriatic arthritis) and placebo with more than 100 patients in each group with active psoriatic arthritis and inadequate response to csDMARDs [10]. At 3 months, an American College of Rheumatology 20 response (assessing improvement of peripheral arthritis) was found in $33 \%$ of the placebo group, $52 \%$ of the adalimumab group and $61 \%$ of the tofacitinib $(2 \times 10 \mathrm{mg})$ group. Clinical response was maintained over 1-year period of treatment, without radiographic structural damage progression in more than $90 \%$ of the patients. Another randomized controlled trial (RCT) evaluated two dosages of tofacitinib versus placebo (more than 130 patients in each group) in active psoriatic arthritis with inadequate response to a TNF inhibitor [11]. At 3 months, an American College of Rheumatology 20 response was found in $49 \%$ of the patients with the highest tofacitinib dosage versus $23 \%$ in the placebo group ( $\mathrm{p}$ 0.0001).

In axial radiographic SpA (ankylosing spondylitis), tofacitinib was evaluated in a Phase II placebo-controlled study [12]. With ASAS 20 as primary outcome measure at week 12, more than 200 patients were randomized (placebo, 2, 5 or $10 \mathrm{mg}$ tofacitinib two-times a day). In a Bayesian model, primary outcome was achieved for the $10 \mathrm{mg}$ group (ASAS 20 responders: 64 vs $40 \%$ in the placebo group), with superiority of all tofacitinib groups over placebo in observed secondary clinical outcomes. In parallel, a significant reduction in MRI inflammatory scores of spine and sacroiliac joints was observed in the 5 and $10 \mathrm{mg}$ tofacitinib groups. No particular safety concern rose over 16 weeks. These findings may support the results of JAK pathways inhibition by tofacitinib in inflammation and new bone formation in animal models of SpA [13].

Nilotinib is a tyrosine kinase (c-kit) inhibitor inducing apoptosis of mast cells. A small proof-of-concept, randomized double-blind, controlled study evaluated nilotinib versus placebo over 12 weeks in peripheral SpA $(n=13)$ and axial SpA $(n=15)$ [14]. In peripheral SpA, nilotinib demonstrated efficacy compared with placebo in reducing significantly synovial inflammation (disease activity, histopathology on synovial biopsies, reduction of c-kit and pro-inflammatory cytokines like IL-6) and C-reactive protein. At the opposite, in axial SpA, no improvement in clinical parameters or serum inflammation markers was found. These results may suggest different implication of this signaling pathway in different phenotypic subtypes of SpA.

Taking these results into consideration, the ts $\mathrm{D}$ MARDs evaluated, demonstrated interesting results in some subsets of patients with SpA. But, to date, the studies are scarce, and the results need to be confirmed. In a second step, it will be necessary to have results of comparative head-to-head studies with standard treatments, namely methotrexate in peripheral SpA and TNF inhibitors in axial as well as peripheral SpA. This kind of result will help to define the best position for tsDMARDs in the treatment strategy of this disease. In this perspective, the use of apremilast has been proposed at several steps in the treatment of psoriatic arthritis, according to the clinical phenotype of the disease in some recommendations [15].

Some positive points should be underlined. All these tsDMARDs are small molecules, orally administered and in consequence simpler in everyday practice than biodrugs. Compared with biodrugs, production processes are less complicated, that may raise the hope of lower costs. Due to their nonproteinic structure, tsDMARDs are not prone to induce immunogenicity; in consequence, the potential questions related to immunogenicity of biodrugs in the treatment of chronic inflammatory rheumatic disorders [16] are out of debate.

At the other hand, some questions need to be answered. The first one is the long-term safety profile of these drugs in SpA. The short-term studies discussed above and the data from the use in other conditions (JAK inhibitors in rheumatoid arthritis for example) seem reassuring to date. Another question is the different impact on disease phenotype. In the 
available data, Apremilast and tyrosine kinase inhibitor demonstrated efficacy on peripheral arthritis but not on axial symptoms, contributing to the discussion of potential different mechanisms of inflammation involved in each of these presentations of the disease. This may represent a matter of further investigation on immune pathological mechanisms of the disease, and should be taken into account in the future use of these drugs. Extra-articular manifestations are part of the disease spectrum and the effect of a new therapeutic option upon these features is of importance and may be incorporated in a decision tree. We will need to know if tsDMARDs are effective upon uveitis or gut inflammation for example.

\section{References}

Papers of special note have been highlighted as:

- of interest; $\bullet$ of considerable interest

1 Taurog JD, Chhabra A, Colbert RA. Ankylosing spondylitis and axial spondyloarthritis. N. Engl. J. Med. 374(26), 2563-2574 (2016).

2 Wendling D, Claudepierre P, Prati C, Dougados M. Spondyloarthritis: a concept or a disease? Joint Bone Spine 82(6), 387-389 (2015).

3 Wendling D, Lukas C, Paccou J et al. Recommendations of the French Society for Rheumatology (SFR) on the everyday management of patients with spondyloarthritis. Joint Bone Spine 81(1), 6-14 (2014).

4 Smolen JS, van der Heijde D, Machold KP, Aletaha D, Landewé R. Proposal for a new nomenclature of diseasemodifying antirheumatic drugs. Ann. Rheum. Dis. 73(1), 3-5 (2014).

5 Abdulrahim H, Thistleton S, Adebajo AO, Shaw T, Edwards C, Wells A. Apremilast: a PDE4 inhibitor for the treatment of psoriatic arthritis. Expert Opin. Pharmacother. 16(7), 1099-1108 (2015).

6 Souto A, Gómez-Reino JJ. Apremilast for the treatment of psoriatic arthritis. Exp. Rev. Clin. Immunol. 11(12), 1281-1290 (2015).

7 Pathan E, Abraham S, Van Rossen E et al. Efficacy and safety of apremilast, an oral phosphodiesterase 4 inhibitor, in ankylosing spondylitis. Ann. Rheum. Dis. 72(9), 1475-1480 (2013).

8 Celgene Corporation. Study of apremilast to treat subjects with active ankylosing spondylitis (POSTURE). In: ClinicalTrials.gov National Library of Medicine, Bethesda, MD, USA (2000) (cited 29 October 2015). Available from: NLM Identifier: NCT01583374.

9 Roskoski R Jr. Classification of small molecule protein kinase inhibitors based upon the structures of their drug-enzyme complexes. Pharmacol. Res. 103(1), 26-48 (2016).

10 Mease PJ, Hall S, FitzGerald $\mathrm{O}$ et al. Efficacy and safety of tofacitinib, an oral janus kinase inhibitor, or adalimumab in patients with active psoriatic arthritis and an inadequate
Due to the large number and diversity of intracellular kinases, the family of tsDMARDs may expand, and all the members should be evaluated, to find out the best candidates to increase the armament against the disease.

\section{Financial \& competing interests disclosure}

The authors have no relevant affiliations or financial involvement with any organization or entity with a financial interest in or financial conflict with the subject matter or materials discussed in the manuscript. This includes employment, consultancies, honoraria, stock ownership or options, expert testimony, grants or patents received or pending, or royalties.

No writing assistance was utilized in the production of this manuscript.

response to conventional synthetic dmards: a randomized, placebo-controlled, Phase III trial. Arthritis Rheumatol. 68(Suppl. 10), Abstract 2983 (2016).

11 Gladman DD, Rigby W, Azevedo VF et al. Efficacy and safety of tofacitinib, an oral janus kinase inhibitor, in patients with active psoriatic arthritis and an inadequate response to tumor necrosis factor inhibitors: opal beyond, a randomized, double blind, placebo-controlled, Phase III Trial. Arthritis Rheumatol. 68(Suppl. 10), Abstract 10L (2016).

12 van der Heijde D, Deodhar AA, Wei JC et al. Tofacitinib in patients with ankylosing spondylitis: a Phase II, 16-week, randomized, placebo-controlled, dose-ranging study. Arthritis Rheumatol. 67(Suppl. 10), Abstract 5L (2015).

Full Text: Ann. Rheum. Dis. 2017 doi:10.1136/ annrheumdis-2016-210322 (Epub ahead of print).

- The first study reporting efficacy of kinase inhibition in axial spondyloarthritis.

13 Lories R, De Wilde K, Heritage K et al. Tofacitinib inhibits inflammation and new bone formation in murine spondyloarthritis but does not adversely inhibit normal human MSC function. Arthritis Rheumatol. 67(Suppl. 10), Abstract 984 (2015).

14 Paramarta JE, Turina MC, Noordenbos T et al. A proof-ofconcept study with the tyrosine kinase inhibitor nilotinib in spondyloarthritis. J. Transl. Med. 14(1), 308 (2016).

- A well-documented study illustrating different efficacy of kinase inhibition according to the presentation of the disease.

15 Coates LC, Kavanaugh A, Mease PJ et al. Group for research and assessment of psoriasis and psoriatic arthritis 2015 treatment recommendations for psoriatic arthritis. Arthritis Rheumatol. 68(5), 1060-1071 (2016).

- Experts' recommendations with proposal-of-use of targeted synthetic disease-modifying antirheumatic drugs in the several subtypes of psoriatic arthritis.

16 Wendling D, Verhoeven F, Guillot X, Prati C. Immunogenicity of TNF alpha inhibitors in rheumatology: many questions, enough answers? Expert Opin. Drug Saf. 16(1), 1-3 (2017). 
